\title{
Optical and electronic properties of functionalized pentacene and anthradithiophene derivatives
}

\author{
Oksana Ostroverkhova ${ }^{a}$, Andrew D. Platt ${ }^{a}$, Whitney E. B. Shepherd ${ }^{a}$, \\ Jonathan Day ${ }^{a}$, and John E. Anthony ${ }^{b}$ \\ ${ }^{a}$ Department of Physics, Oregon State University, Corvallis, OR 97331, USA; \\ ${ }^{b}$ Department of Chemistry, University of Kentucky, Lexington, KY 40506, USA
}

\begin{abstract}
The optical, fluorescent, and photoconductive properties of solution-processable functionalized pentacene and anthradithiophene (ADT) derivatives are presented. Considerable fluorescence quantum yields of $\sim 70-75 \%$ and $\sim 40-50 \%$ were observed in several ADT derivatives in toluene solutions and in thin films, respectively. Using conventional wide-field fluorescence microscopy, ADT derivatives were successfully imaged in the polymethylmethacrylate (PMMA) matrix on a single molecule level, at $532 \mathrm{~nm}$ at room temperature. All films exhibited fast charge carrier photogeneration upon $100 \mathrm{fs} 400 \mathrm{~nm}$ excitation and power-law decay dynamics of the transient photocurrent over many orders of magnitude in time. In solution-deposited ADT thin films, effective charge carrier mobilities calculated from the space-charge-limited currents reached $\sim 0.1 \mathrm{~cm}^{2} / \mathrm{Vs}$. In the same films, bulk photoconductive gains of up to 130 were observed at $532 \mathrm{~nm}$ continuous wave (cw) excitation with light intensity of $0.58 \mathrm{~mW} / \mathrm{cm}^{2}$ at the applied electric field of $4 \times 10^{4} \mathrm{~V} / \mathrm{cm}$. Effects of metal-organic interfaces on the dark current and transient and cw photocurrent are also discussed.
\end{abstract}

Keywords: organic semiconductors, fluorescence, photoconductivity, space-charge-limited current, single-molecule spectroscopy

\section{INTRODUCTION}

Organic semiconductors have been investigated as an alternative to conventional inorganic semiconductors due to their low cost, ease of fabrication, and tunable properties. ${ }^{1}$ Applications envisioned for organic semiconductors include xerography, thin-film transistors, light-emitting diodes, lasers, solar cells and photorefractive devices. ${ }^{2-4}$ Small-molecular-weight solution-processable materials that can be cast into high-performance (photo)conductive thin films are of particular technological interest.

Functionalized anthradithiophene (ADT) and pentacene derivatives have attracted considerable attention due to their high charge carrier mobility, ${ }^{5-7}$ photoconductivity ${ }^{8-13}$ and luminescence. ${ }^{5,12,14,15}$ In particular, charge carrier (hole) mobilities of over $1.2 \mathrm{~cm}^{2} / \mathrm{Vs}$ have been observed in solution-deposited films of pentacene functionalized with triisopropylsilylethynyl (TIPS) groups and of fluorinated ADT functionalized with triethylsilylethynyl (TES) side groups. ${ }^{6,16}$ In addition, in similar films, fast charge carrier photogeneration and non-thermallyactivated charge transport on picosecond time scales after photoexcitation, have been reported. ${ }^{8-10}$ In films of conjugated functionalized pentacene dimers, photoconductive gains of over 10 have been observed. ${ }^{12}$ Finally, high photoluminescence quantum yields (QYs) of $72 \%$ and $76 \%$ have been obtained in dioxolane-substituted pentacene derivatives in toluene solution and when used as guest molecules (at $0.25 \mathrm{~mol} \%$ concentration) in $\mathrm{Alq}_{3}$ films, respectively. ${ }^{14}$

Slight chemical modifications of the side groups of both ADT and pentacene derivatives lead to considerable changes in molecular packing, ${ }^{5}$ which affects electronic and optical properties of thin films. Additional changes in these properties may be produced by functionalization of the core of the molecule. In this paper, we report on the optical, fluorescent, and (photo)conductive properties of several functionalized ADT and pentacene derivatives.

Further author information: (Send correspondence to O.O.)

O.O: E-mail: oksana@science.oregonstate.edu, Telephone: 1-541-737-1679

J.E.A.: E-mail: anthony@uky.edu, Telephone: 1-859-257-8844 

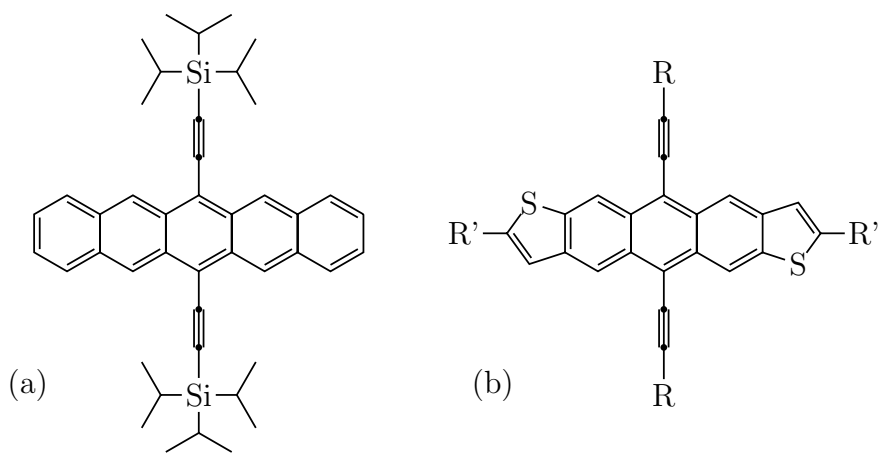

Figure 1. (a) Pentacene functionalized with side groups of $\operatorname{Si} i$ - $\operatorname{Pr}_{3}$ (TIPS) and (b) anthradithiophene functionalized with side groups of $\mathrm{R}=\mathrm{SiEt}_{3}$ (TES) or $\mathrm{Si}_{-}-\mathrm{Pr}_{3}$ (TIPS) and $\mathrm{R}^{\prime}=\mathrm{F}$ or $\mathrm{CN}$.

Our goals are (i) to establish influence of modifications of the core and of the side groups of the molecule on the photophysical properties of molecules in solutions and thin films, and on the (opto)electronic properties of thin films; (ii) to probe effects of intermolecular interactions into optical, fluorescent, and (photo)conductive properties of thin films; and (iii) to identify contributions of processes at metal-organic interfaces into a (photo)conductive response of thin films.

\section{EXPERIMENTAL}

\subsection{Materials and sample preparation}

The synthesis of functionalized pentacene (TIPS-pentacene) and ADT derivatives (ADT-TIPS(TES)-F(CN)) used in our studies (Fig. 1) has been reported elsewhere. ${ }^{15,17}$

Stock solutions of functionalized ADT and pentacene derivatives were prepared at $\sim 1 \%$ by weight in toluene. For quantum yield measurements, solutions were prepared by dilution of stock solutions to $\sim 10^{-5} \mathrm{M}$. For measurements of fluorescence spectra in the solid state, but without effects of significant intermolecular interaction, the mixtures of a $10^{-5} \mathrm{M}$ solution of molecules under study and $1 \mathrm{wt} \%$ of polymethylmethacrylate (PMMA) in toluene were prepared and drop-cast onto a glass substrate at $\sim 60{ }^{\circ} \mathrm{C}$. For all other measurements, films with thickness of $0.5-2 \mu \mathrm{m}$ were prepared by drop-casting stock solutions onto glass substrates at $\sim 60{ }^{\circ} \mathrm{C}$.

For measurements of dark currents and photoresponse, glass substrates were prepared by photolithographic deposition of $5 \mathrm{~nm} / 50 \mathrm{~nm}$ thick $\mathrm{Cr} / \mathrm{Au}$ or $100 \mathrm{~nm}$ thick aluminum (Al) electrode pairs. Each pair consisted of 10 interdigitated finger pairs, with $1 \mathrm{~mm}$ finger length, $25 \mu \mathrm{m}$ finger width and $25 \mu \mathrm{m}$ gaps between the fingers of opposite electrodes. Films were drop-cast onto the interdigitated regions. The coplanar electrode geometry, with 25 or $50 \mu \mathrm{m}$ gap and $1 \mathrm{~mm}$ channel width, was also used, in addition to interdigitated geometry. With the exception of ADT-TIPS-CN, materials investigated here formed polycrystalline films when solution deposited, as confirmed by x-ray diffraction and transmission electron microscopy (TEM). At least 10 samples of each material were studied.

\subsection{Optical Properties}

Optical absorption spectra were measured using a halogen lamp and a fiber-coupled Ocean Optics USB2000 spectrometer. Absorbance $A$ was calculated from the incident $\left(I_{0}\right)$ and transmitted $(I)$ beam intensities as $A=-\log \left(I / I_{0}\right)$. Reflection losses were taken into account by referencing with respect to cuvettes with pure solvent or clean glass substrates for solution and film measurements, respectively.

Solution emission spectra were acquired in a custom fluorescence measurement setup with laser excitation at wavelengths of either $400 \mathrm{~nm}$ (Ti:Sapphire laser from KM Labs frequency-doubled with a beta-barium borate (BBO) crystal), $532 \mathrm{~nm}$ (Nd:YVO 4 laser from Coherent, Inc.), $633 \mathrm{~nm}$ (HeNe laser), or $655 \mathrm{~nm}$ (diode laser). Emitted photons were collected using a parabolic mirror and detected with a fiber coupled spectrometer (Ocean Optics USB2000) calibrated against a $3100 \mathrm{~K}$ black-body emitter. Absorption of solutions was measured using 
a standard $1 \mathrm{~cm}$ path length quartz cuvette with a halogen light source fiber-optically delivered to the sample holder and spectrometer. Fluorescence quantum yields (QYs) in solution were referenced against standards with known quantum yields and corrected for differences in optical density and solvent refractive index. ${ }^{18}$ The ADT derivatives were measured against rhodamine $6 \mathrm{G}$ in ethanol $\left(\Phi_{\mathrm{f}}=0.95\right)$ and DCDHF-N-6 in toluene $\left(\Phi_{\mathrm{f}}=0.85\right) .{ }^{19}$ The QY of TIPS pentacene solution was measured against rhodamine $6 \mathrm{G}$ in ethanol and Alexa Fluor 647 in a phosphate buffer solution $\left(\mathrm{pH} 7.2, \Phi_{\mathrm{f}}=0.33\right) .{ }^{20}$ The QYs in films were estimated using DCDHFN-6 in PMMA $\left(\Phi_{\mathrm{f}}=0.98\right)^{19}$ as a reference and assuming a value of 1.7 for the index of refraction. The detection limit of the setup was estimated to be at $\mathrm{QY} \approx 0.5 \%$.

Fluorescence lifetime measurements were performed using a frequency-doubled mode-locked Ti:Sapphire laser with a repetition rate of $93 \mathrm{MHz}$ picked at $9.3 \mathrm{MHz}$ using a home-built pulse picker (based on a $\mathrm{TeO}_{2}$ acoustooptic modulator from NEOS) and pulses $80 \mathrm{fs}$ in length as the excitation source. A single-photon avalanche photodiode (SPAD - Molecular Photonic Devices) was used in conjunction with a time-correlated single-photon counter (TCSPC) data analysis board (PicoQuant TimeHarp 200) for detection. The instrument response function (IRF) ( $\sim 200 \mathrm{ps})$ was recorded using scattered light from an etched microscope slide.

\subsection{Single-molecule-level fluorescence imaging}

Samples for fluorescence imaging at the single-molecule level were prepared from stock solutions of $1 \%$ by weight PMMA (75,000 m.w.) in toluene. ADT-TES-F was doped into the solution at the level of $10^{-10}$ per PMMA polymer. This solution was then spun coat onto clean glass coverslips at $2000 \mathrm{rpm}$ for $55 \mathrm{~s}$.

The samples were imaged with an Olympus IX71 inverted microscope with a 100X UPlanApo objective under wide-field $532 \mathrm{~nm} \mathrm{cw}$ illumination. The fluorescence was detected by an Andor iXon DU-897 EMCCD camera.

\subsection{Dark current and transient and cw photocurrent measurements}

For transient photoconductivity measurements, an amplified Ti:Sapphire laser $(800 \mathrm{~nm}, 100 \mathrm{fs}, 1 \mathrm{kHz})$ was used in conjunction with a frequency-doubling BBO crystal to excite the samples. Voltage was supplied by a Keithley 237 source-measure unit, and light pulse-induced transient photocurrent was measured with a $50 \Omega$ load by a $50 \mathrm{GHz}$ CSA8200 digital sampling oscilloscope. ${ }^{10,11,15,21}$ Average electric field $E$ was calculated as $E=V / L$, where $V$ is the applied voltage, and $L$ is the gap between the electrodes. For dark current and cw photocurrent measurements, the Keithley 237 source-measure unit was used to measure current through the sample in the absence and in the presence of cw photoexcitation with a Nd:YVO 4 laser at $532 \mathrm{~nm}$. The cw photocurrent was calculated as the difference between the two.

\section{RESULTS AND DISCUSSION}

\subsection{Optical properties in solution}

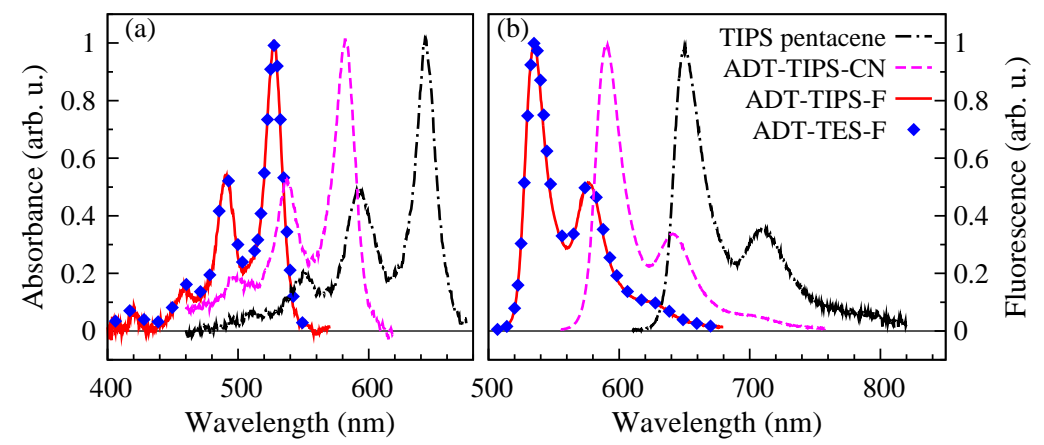

Figure 2. Normalized absorption (a) and fluorescence (b) spectra of toluene solutions of ADT-TES-F, ADT-TIPS-F, ADT-TIPS-CN, and TIPS-pentacene. 
Table 1. Optical properties of functionalized pentacene and ADT derivatives in solution.

\begin{tabular}{c|cccc}
\hline & $\begin{array}{c}\lambda_{\text {abs }}{ }^{a} \\
(\mathrm{~nm})\end{array}$ & $\begin{array}{c}\lambda_{\mathrm{em}}{ }^{b} \\
(\mathrm{~nm})\end{array}$ & $\Phi$ & $\begin{array}{c}\tau \\
(\mathrm{ns})\end{array}$ \\
\hline ADT-TES-F & 528 & 536 & 0.70 & 9.4 \\
ADT-TIPS-F & 528 & 536 & 0.72 & 9.1 \\
ADT-TIPS-CN & 582 & 590 & 0.76 & 12.7 \\
TIPS pentacene & 643 & 650 & 0.75 & 11.8
\end{tabular}

${ }^{a}$ Wavelength of maximal absorption, which corresponds to $0 \rightarrow 0$ transition (Fig. 2(a)).

${ }^{b}$ Wavelength of maximal emission, which corresponds to $0 \rightarrow 0$ transition (Fig. 2(b)).

Figure 2 shows optical and fluorescent properties of several functionalized ADT and TIPS pentacene derivatives in toluene solution. In solution, optical properties were determined primarily by their molecular core and were not affected by TIPS or TES side-groups, which resulted in identical spectra of, for example, ADT-TES-F and ADT-TIPS-F in Fig. 2 or TIPS and TES pentacene (only TIPS pentacene data are shown). Spectra of ADTTIPS-CN and TIPS pentacene in solution were both red-shifted with respect to those of ADT-TIPS(TES)-F. Small Stokes shifts of $<10 \mathrm{~nm}$, observed in all solutions, are due to rigidity of the molecular core. ${ }^{12,22,23}$ Fluorescence lifetime decay of solutions was well described by a single-exponential function $(\sim \exp [-t / \tau]$, where $\tau$ is the fluorescence lifetime). ${ }^{15,24}$ ADT-TIPS-F and ADT-TES-F derivatives exhibited similar lifetimes $(\tau)$ of $\sim 9 \mathrm{~ns}$ and high fluorescence quantum yields (QYs) $(\Phi)$ of $\sim 70 \%$ in toluene. Solutions of ADT-TIPS-CN and TIPS pentacene showed longer lifetimes ( 12-12.5 ns) and QY $\sim 75 \%$ (Table 1$).{ }^{15}$

\subsection{Optical properties in film}

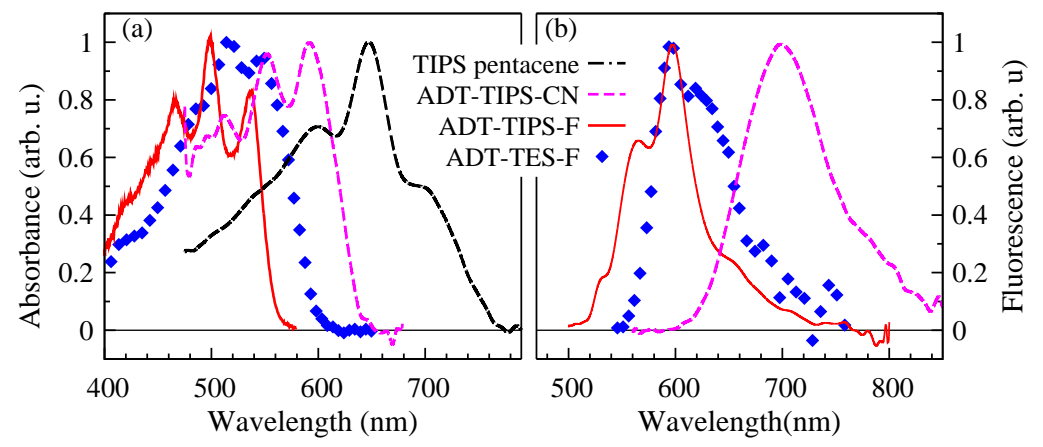

Figure 3. Normalized optical absorption (a) and fluorescence (b) spectra of films.

Optical absorption spectra of films (Fig. 3(a)) exhibited a redshift, or displacement $\Delta$, with respect to those in solutions, due to enhanced Coulomb interaction of the molecule with its surrounding and exchange interaction between translationally equivalent molecules. ${ }^{25}$ In general, $\Delta$ depends on the molecular-orbital overlap and on the structure and morphology of the film. ${ }^{9,15}$ In contrast to identical absorption and fluorescence spectra of ADTTIPS-F and ADT-TES-F in solution, those of corresponding films were considerably different (Fig. 3), which we attribute to differences in packing of these molecules in the solid and in film crystallinity. ${ }^{17}$ In particular, ADT-TIPS-F and ADT-TES-F films exhibited displacements $\Delta$ of $\sim 320 \mathrm{~cm}^{-1}$ and $\sim 760 \mathrm{~cm}^{-1}$, respectively. Although $\Delta$ exhibited sample-to-sample variation, it was always larger in ADT-TES-F films compared to ADTTIPS-F ones, indicative of a higher degree of exciton delocalization in ADT-TES-F films. Also redshifted were fluorescence spectra of ADT-TES-F with respect to those of ADT-TIPS-F films (Fig. 3(b)). Vibronic bands in both absorption and fluorescence spectra were broader in films, as compared to solutions, with relative intensities of the bands varied depending on the film thickness and morphology (Fig. 4). Regardless of the film thickness, ADT-TIPS-F films showed a more pronounced vibronic structure of the fluorescence spectra than ADT-TES-F or ADT-TIPS-CN. The differences observed in fluorescence spectra of films compared to those in solutions are due to intermolecular interactions leading to a formation of molecular aggregates (whose properties depend on the 
degree of molecular order, size, and intermolecular coupling). ${ }^{26-28}$ This is further supported by our observations that the fluorescence spectra of molecules under study embedded at low concentrations in the PMMA matrix (Fig. 4) yielded spectra identical to those of solutions in Fig. 1(b), as expected from non-interacting molecules. ${ }^{29}$ Although all materials studied could be prone to aggregate formation due to their $\pi$-stacking properties, ${ }^{5,17}$ fluorescent properties of aggregates significantly depended on the material. For example, at room temperature, thin films of ADT-TIPS-F and ADT-TES-F were highly fluorescent, with QYs of at least $40-50 \%$ depending on the film thickness and morphology. (These values represent a lower limit, since effects of self-absorption were significant in even the thinnest of our films). In contrast, fluorescence in TIPS pentacene films was considerably weaker (QYs of $<0.5 \%$ ). In all ADT films studied, QYs were strongly temperature-dependent, as described in detail in Refs. 15 and 24.

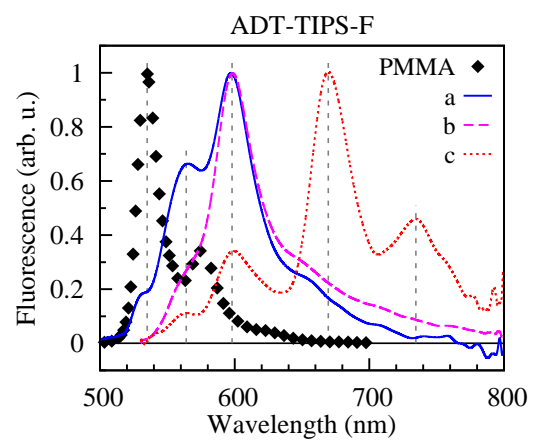

Figure 4. Fluorescence spectra of ADT-TIPS-F dispersed in PMMA in low concentration, and of ADT-TIPS-F films of different thicknesses and crystallinity. The samples a and b are morphologically distinctive from sample c. The samples $\mathrm{a}, \mathrm{b}$, and $\mathrm{c}$ have optical densities of $2.2,2.1$, and 3.4, respectively, at the wavelength of maximal absorption.

In all materials studied, the fluorescence decay dynamics in films were faster than those in solution and could be described by a bi-exponential function $\left(\sim a_{1} \exp \left[-t / \tau_{1}\right]+a_{2} \exp \left[-t / \tau_{2}\right]\right.$, where $\tau_{1(2)}$ and $a_{1(2)}$ are shorter (longer) lifetimes and their relative amplitudes, respectively, where $a_{1}+a_{2}=1$ ), characteristic of molecular aggregates. ${ }^{26}$ Both $\tau_{1}$ and $\tau_{2}$ were shorter than lifetimes $\tau$ measured in solutions of the same molecules, and the weighted average lifetimes in films, $\tau_{\mathrm{av}}=a_{1} \tau_{1}+a_{2} \tau_{2}$, were typically on the order of $0.4-3 \mathrm{~ns}$ at room temperature, depending on the material, and varied with film quality (e.g. from 1.1 to $2.5 \mathrm{~ns}$ in ADT-TIPS-F films). ${ }^{15,24}$

\subsection{Properties of individual molecules}

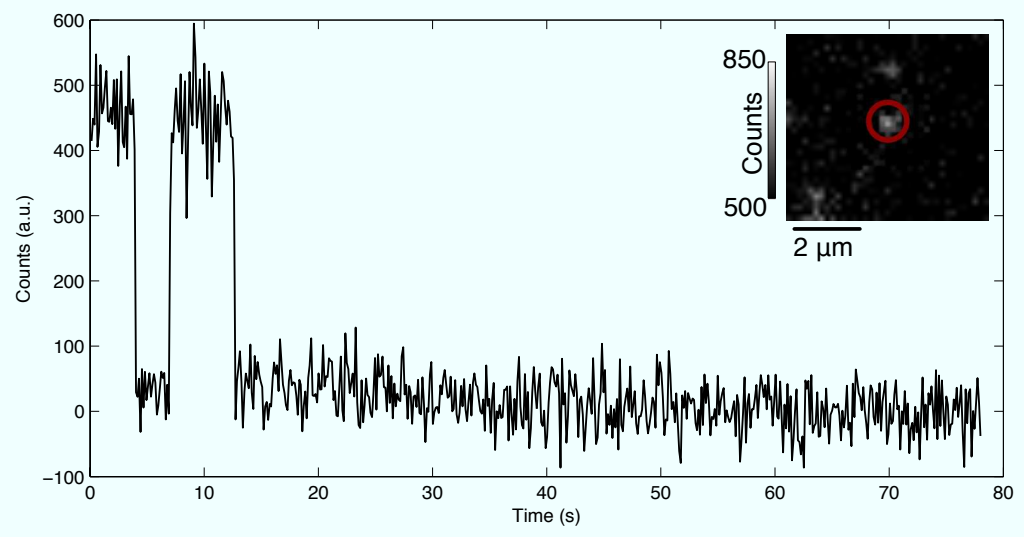

Figure 5. Blinking behavior of an ADT-TES-F molecule embedded in a PMMA matrix (labeled in inset). Data was taken under $700 \mu \mathrm{W}$ of $532 \mathrm{~nm} \mathrm{cw}$ illumination. Single-step blinking and photobleaching are characteristic features of single-molecule behavior. 
As seen from Fig. 5, which shows a typical time dependence of the fluorescence photon count obtained from an individual ADT-TES-F molecule in a PMMA matrix, ADT-TES-F has been successfully imaged at the single molecule level at room temperature. Signal-to-noise ratios of over 10:1 were obtained at moderate excitation levels. ADT-TES-F exhibited good stability as a single molecule fluorophore, comparable to DCDHF-N-6, which is one of the best fluorophores utilized in single-molecule fluorescence spectroscopy. ${ }^{19}$ This opens up new possibilities to study charge- and energy-transfer processes in these materials at nanoscales.

\section{4 (Photo)conductive properties}

\subsubsection{Transient photocurrent}

Upon excitation with $400 \mathrm{~nm} 100$ fs pulses, all samples showed fast photoresponse, with the rise time of 30-40 ps, limited by the time resolution of our setup (inset of Fig. 6), ${ }^{10}$ which indicates fast charge carrier photogeneration in these materials. ${ }^{8,21,30}$ Transient photocurrents obtained in ADT-TIPS-F and ADT-TES-F films on $\mathrm{Au}$ electrodes upon excitation with $400 \mathrm{~nm}$ pulses at the fluence of $\sim 5 \mu \mathrm{J} / \mathrm{cm}^{2}$ and average electric field $E$ of $4 \times 10^{4} \mathrm{~V} / \mathrm{cm}$ are shown in Fig. 6. In all polycrystalline films, transient photocurrents exhibited behavior previously observed in several organic crystals: ${ }^{8,9,30}$ fast initial decay, most likely due to initial carrier trapping and recombination, followed by a slow component that can be fitted with a power-law function $\left(I_{\mathrm{ph}} \sim t^{-\beta}\right)$ with $\beta \sim 0.2-0.6$, depending on the sample and on the material, over at least three orders of magnitude (see, for example, inset of Fig. 8(a)). ${ }^{10,15,21,29}$ Among ADT-TIPS-F, ADT-TES-F, and TIPS pentacene samples, the decay of the transient photocurrent was, on average, slowest in ADT-TES-F, followed by ADT-TIPS-F and TIPS pentacene, when measured under the same conditions. Power-law exponents $\beta$ describing the transient photocurrent decay dynamics on time scales from sub-ns to at least tens of $\mu$ s after photoexcitation were also slightly different, with $\beta$ of $\sim 0.2-0.4$ in ADT-TES-F and of $\sim 0.4-0.6$ in ADT-TIPS-F and TIPS pentacene films. ${ }^{10,11,21}$ From the peak of the transient photocurrent $\left(I_{\mathrm{ph}, \max }\right)$, a product of intrinsic charge carrier mobility $\mu$ and photogeneration efficiency $\eta$ was calculated using $\mu \eta=I_{\mathrm{ph}, \max } /\left(e N_{\mathrm{ph}} E d\right)$, where $N_{\mathrm{ph}}$ is the number density of absorbed photons per pulse, $E$ is the electric field, $e$ is the charge of the electron, and $d$ is the channel width. The $\mu \eta$ products were comparable in all three materials, yielding values between 0.01 and $0.025 \mathrm{~cm}^{2} / \mathrm{Vs}$ at $1.2 \times 10^{4} \mathrm{~V} / \mathrm{cm}$ (Table 2). Here $\eta$ includes trapping and recombination that occurred during the first tens of picoseconds after photoexcitation, not resolved in our experiments, and therefore, $\eta<\eta_{0}$, where $\eta_{0}$ is the initial photogeneration efficiency. If intrinsic mobilities in our materials are on the order of $1 \mathrm{~cm}^{2} / \mathrm{Vs}$, then the lower limit for the initial photogeneration efficiency $\eta_{0}$ is $\sim 1-2 \%$, which is comparable to that of $5 \%$ estimated from the transient photocurrents measured in tetracene single crystals. ${ }^{31}$

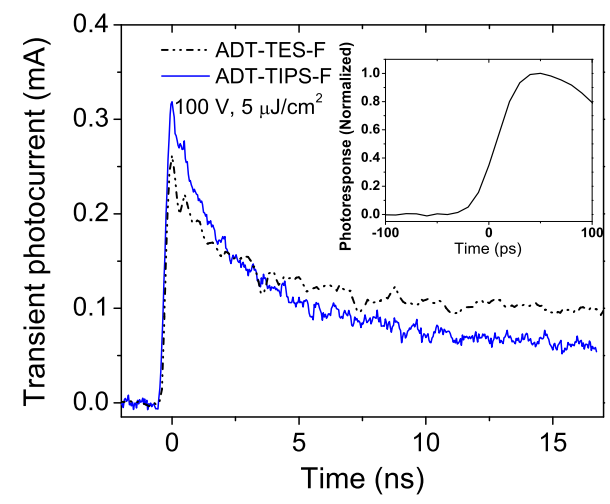

Figure 6. Transient photocurrent obtained in ADT-TIPS-F and ADT-TES-F films under 400-nm 100 fs excitation. Inset illustrates sub- 40 ps rise dynamics of the photocurrent, limited by the time resolution of our setup. 


\subsubsection{Dark current}

Space-charge-limited currents (SCLCs) were observed in the ADT-TIPS-F, ADT-TES-F and TIPS pentacene films on Au electrodes. ${ }^{11,15}$ Effective charge carrier mobilities $\left(\mu_{\mathrm{eff}}\right)$ were calculated from the slope of the fits of the dark current as a function of applied voltage squared. In the case of planar electrode geometry used here, the current flows along a layer of unknown thickness, and there is no analytical solution for the relationship between SCLC (linear) density $\left(j=I_{\mathrm{d}} / d\right.$, where $I_{\mathrm{d}}$ is the dark current and $d$ is the channel width) and voltage $(V)$ in a film of finite thickness. ${ }^{11,32}$ In the approximation of the infinitely thin film, valid when the film thickness is much lower than the gap width $(L)$ between the electrodes (which is the case here), $j=2 \mu_{\text {eff } \epsilon \epsilon_{0}} V^{2} /\left(\pi L^{2}\right)$, where $\epsilon$ is a relative dielectric constant of the film and $\epsilon_{0}$ is the dielectric permittivity of vacuum. SCLC effective mobilities $\left(\mu_{\text {eff }}\right)$, which represent a lower bound of charge carrier mobilities in these films, showed sample-tosample variation, especially significant in the case of ADT-TIPS-F and TIPS pentacene (Table 2). On average, however, $\mu_{\text {eff }}$ in ADT-TES-F was at least a factor of $\sim 3$ higher than that in ADT-TIPS-F, and a factor of $\sim 7$ higher than in TIPS pentacene films. A much lower current was observed in ADT-TIPS-CN films, which was a result of both poor hole injection from $\mathrm{Au}$ contact due to a relatively high barrier of $\sim 0.45 \mathrm{eV} \mathrm{Au}$ forms with ADT-TIPS-CN and the amorphous nature of the film. The effective mobility $\mu_{\text {eff }}=\mu \theta$, where $\theta$ is the ratio between free and trapped charge carriers $(\theta \leq 1)$ is lower than the intrinsic mobility $\mu$, since most likely the trapfree limit has not been achieved in our samples. ${ }^{33}$ Indeed, although $\mu_{\text {eff }}$ values of $0.033-0.092 \mathrm{~cm}^{2} / \mathrm{Vs}$ obtained in ADT-TES-F films were similar to those obtained in ADT-TES-F TFTs with channel lengths similar to ours (i.e. $25 \mu \mathrm{m}),{ }^{7}$ mobilities of over $1.5 \mathrm{~cm}^{2} /$ Vs and $6 \mathrm{~cm}^{2} /$ Vs have been achieved in short-channel ADT-TES-F films spin-coated on the Au electrodes treated with pentafluorobenzenethiol ${ }^{6,17}$ and in ADT-TES-F single crystals, ${ }^{34}$ respectively. Therefore, $\theta$ is below $\sim 0.015$ in our ADT-TES-F films. It is lower yet in TIPS pentacene films, since $\mu_{\text {eff }}$ values of $0.002-0.007 \mathrm{~cm}^{2} / \mathrm{Vs}$ obtained in our SCLC measurements are much lower than $1.2 \mathrm{~cm}^{2} / \mathrm{Vs}$ observed in TIPS pentacene TFTs, ${ }^{16}$ indicative of high trap density in our TIPS pentacene films.

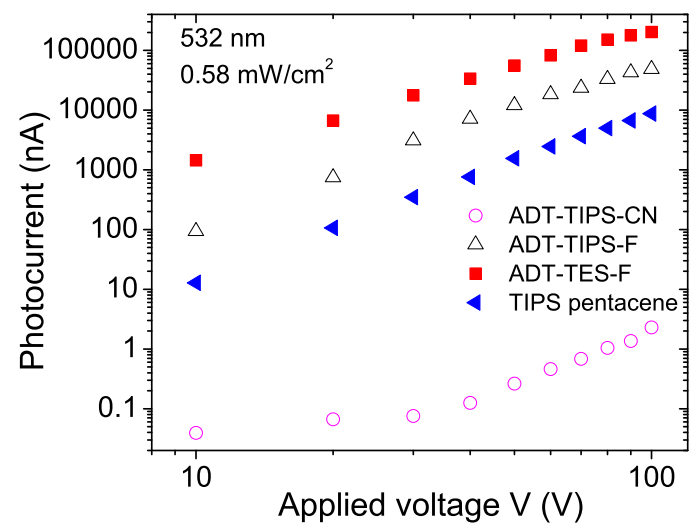

Figure 7. Cw photocurrent obtained in ADT-TES-F, ADT-TIPS-F, ADT-TIPS-CN, and TIPS pentacene films on interdigitated $\mathrm{Au}$ electrodes as a function of applied voltage.

\subsubsection{Cw photocurrent}

Figure 7 shows cw photocurrent obtained at $532 \mathrm{~nm}$ excitation of ADT-TIPS-F, ADT-TES-F, ADT-TIPS-CN, and TIPS pentacene films on interdigitated Au electrodes. In all samples, cw photocurrent measured at light intensity of $0.58 \mathrm{~mW} / \mathrm{cm}^{2}$ was higher than the dark current. Especially strong cw photoresponse was observed in best ADT-TES-F films, with photocurrents of over $200 \mu \mathrm{A}$ at the average electric field of $4 \times 10^{4} \mathrm{~V} / \mathrm{cm}$ at low light intensities (Fig. 7). This corresponds to linear photocurrent densities $\left(j_{\mathrm{cw}}=I_{\mathrm{cw}} / d\right)$ of over $0.1 \mathrm{~mA} / \mathrm{cm}$, which, under assumption that photons absorbed throughout the entire thickness of a $1 \mu \mathrm{m}$ thick film may contribute to 
Table 2. (Photo)conductive properties of functionalized pentacene and anthradithiophene derivatives. ${ }^{a}$

\begin{tabular}{rccc}
\hline Name & $\begin{array}{c}\mu_{\text {eff }}^{a, b} \\
\left(\mathrm{~cm}^{2} / \mathrm{Vs}\right)\end{array}$ & $\begin{array}{c}\mu \eta^{a, c} \\
\left(\mathrm{~cm}^{2} / \mathrm{Vs}\right)\end{array}$ & $G^{a, d}$ \\
\hline ADT-TES-F & $0.033-0.092$ & $0.02-0.025$ & $70-130$ \\
ADT-TIPS-F & $0.002-0.029$ & $0.018-0.025$ & $16-30$ \\
TIPS pentacene & $0.002-0.007$ & $0.01-0.022$ & $9-28$
\end{tabular}

${ }^{a}$ Range of numbers represents variation in values obtained in 10 samples of each material.

${ }^{b}$ Calculated from SCLC data at room temperature.

${ }^{c}$ Calculated from the peak of the transient photocurrent obtained in films on $\mathrm{Al}$ electrodes at the average electric field of $1.2 \times 10^{4} \mathrm{~V} / \mathrm{cm}$ at room temperature.

${ }^{d}$ Calculated from the cw photocurrent obtained in films on $\mathrm{Au}$ electrodes as the number of photogenerated carriers per number of absorbed photons at the average electric field of $1.2 \times 10^{4} \mathrm{~V} / \mathrm{cm}$ at $0.58 \mathrm{~mW} / \mathrm{cm}^{2}$ at $532 \mathrm{~nm}$ at room temperature.

the photocurrent, yields (area) current densities $\left(J_{\mathrm{cw}}\right)$ of over $1 \mathrm{~A} / \mathrm{cm}^{2}$. If photoconductivity $\left(\sigma_{\mathrm{ph}}\right)$ is calculated

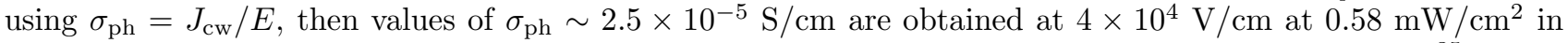
ADT-TES-F, which are considerably higher than those reported in conductive polymers such as PPV ${ }^{35}$ and in unsubstituted pentacene films or tetracene crystals under cw excitation. ${ }^{31,36,37}$ ADT-TIPS-CN films showed the weakest cw photoresponse (Fig. 7), both due to a non-injecting nature of the Au/ADT-TIPS-CN contact ${ }^{11}$ and amorphous nature of the ADT-TIPS-CN film.

Photoconductive gain $G$ was calculated from the cw photocurrents, absorption coefficients, and light intensity as the ratio between the number of carriers flowing in the film and the number of absorbed photons. ${ }^{12,13}$ In the case of hole-transporting materials and hole-injecting electrodes (such as Au in the case of ADT-TIPS(TES)-F and TIPS pentacene), bulk photoconductive gain $G \approx \eta_{0} \tau_{c} / t_{\mathrm{tr}}$ (where $\tau_{c}$ is the carrier lifetime, and $t_{\mathrm{tr}}$ is the time for the hole to transit through the film), and $G$ can be much larger than the initial photogeneration efficiency $\eta_{0}\left(\eta_{0}<1\right)$. Gain $G$ values that are much larger than unity were indeed observed in fluorinated ADT and TIPS pentacene films, as summarized in Table $2 .{ }^{12,13}$ The highest photoconductive gains, up to 130 at $4 \times 10^{4} \mathrm{~V} / \mathrm{cm}$ at $0.58 \mathrm{~mW} / \mathrm{cm}^{2}$, were achieved in ADT-TES-F films, consistent with highest effective mobility $\mu_{\mathrm{eff}}$ (shorter transit time $t_{\mathrm{tr}}$ ) and longest carrier lifetimes (as observed in SCLC and in the transient photocurrent measurements, respectively) in ADT-TES-F films, as compared to other materials under study. ${ }^{10,11,15}$ The values of $G$ measured in ADT-TES-F films were similar to those in GaN photodetectors at similar light intensity levels and at least an order of magnitude higher than those in unsubstituted pentacene and in functionalized pentacene films. ${ }^{11-13}$

\subsubsection{Influence of organic-metal interfaces on the photocurrent}

Transient photocurrent. Figure 8(a) shows transient photocurrents, normalized at their peak values, measured in ADT-TIPS-F films on $\mathrm{Au}$ and $\mathrm{Al}$ electrodes. Interestingly, the faster decay component was more pronounced in films on $\mathrm{Al}$ electrodes, whereas the slower one was independent of the electrode material and could be described by a power-law function $\left(I_{p h} \sim t^{-\beta}\right)$, as discussed above. ${ }^{11}$ In samples with a $25-\mu$ m gap at voltages above $\sim 30 \mathrm{~V}$, the difference in the peak transient amplitudes for films on Au and films on Al electrodes was comparable to that due to morphology-related sample-to-sample variation ${ }^{10}$ of approximately a factor of 2 (Fig. $8(\mathrm{~b})$ ). The relation between the peak amplitude and applied voltage $\left(I_{p h, \max } \sim V^{\alpha}\right)$ was sampledependent, with $\alpha \sim 1.3-1.8 .^{10}$ Analysis of the behavior of many samples, however, yielded that on average, $\alpha$ in samples with $\mathrm{Al}$ electrodes was higher than in those with $\mathrm{Au}$ electrodes (e.g. $\alpha=1.76 \pm 0.06$ and $1.33 \pm 0.01$ in ADT-TIPS-F films on Al and Au electrodes, respectively, in Fig. 8(b)). Similar dependencies were observed for all electrode geometries, regardless of film-side or glass substrate-side illumination. Since measurements of the photocurrent were performed in the presence of applied electric field, the photoresponse was necessarily measured after dark current flow had been established in the film, or, in other words, after the sample had been pre-conditioned by the dark current. In samples with $\mathrm{Au}$ and $\mathrm{Al}$ electrodes, very different charge injection conditions obtain and, therefore, different pre-conditioning of the samples is realized. In particular, samples with $\mathrm{Au}$ electrodes, in which SCLC regime is established, are expected to have a high density of filled hole traps. 

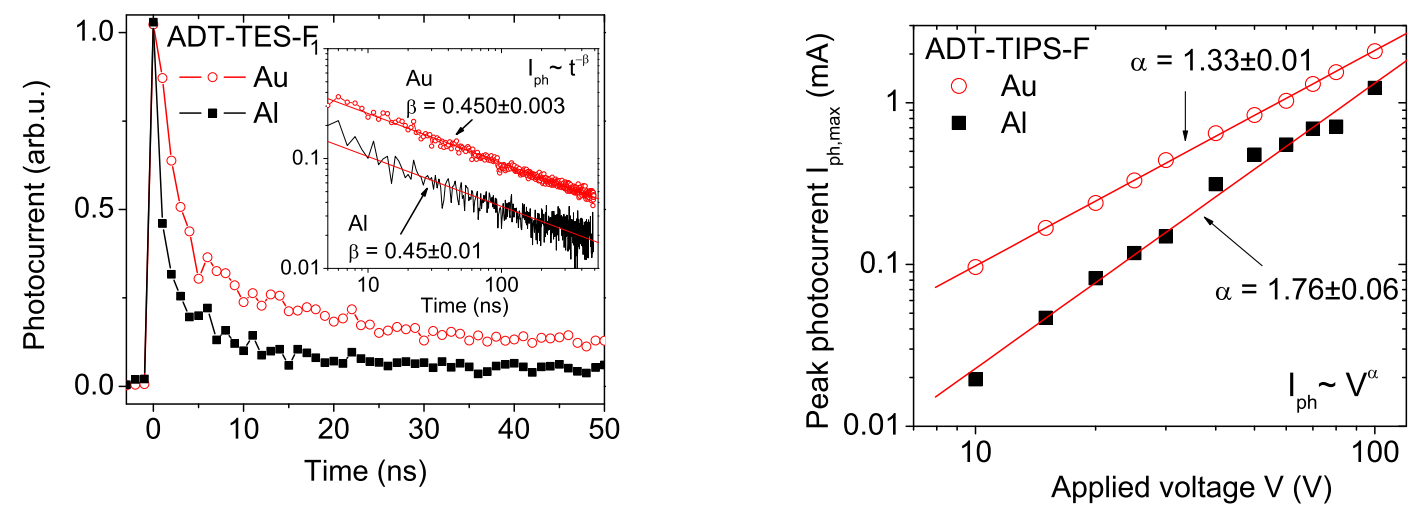

Figure 8. (a) Transient photocurrents, normalized at their peak values, obtained in ADT-TIPS-F films on Au and Al electrodes under the same experimental conditions. Inset: long time-scale dynamics of the same transient photocurrents. Power-law fits $\left(I_{p h} \sim t^{-\beta}\right)$ are also shown. (b) Transient photoresponse peak amplitude $\left(I_{p h, \max }\right)$ as a function of applied voltage obtained in ADT-TIPS-F samples with Al or Au interdigitated electrodes.

In contrast, samples with $\mathrm{Al}$ electrodes are expected to be in a close-to-pristine condition (empty traps) at low voltages, and have partially filled traps at higher voltages, after hole injection via Fowler-Northeim tunneling is enabled. ${ }^{11}$ If fast initial decay is related to bulk material properties and is due to fast trapping of photogenerated holes, then in films on Au electrodes, the density of empty traps is reduced, which in turn reduces initial trapping of the photogenerated holes. ${ }^{11}$ In contrast, most traps in films on $\mathrm{Al}$ electrodes are empty and are readily filled by photogenerated holes, thus leading to a fast initial decay of the photocurrent. This is also consistent with previously observed slowing down of the initial decay of the transient photocurrent upon increasing applied electric field in ADT-TIPS-F films on Al electrodes. ${ }^{10}$

Dark current and cw photocurrent Unlike the amplitudes of transient photocurrent, the cw photocurrents $\left(I_{c w}\right)$ and dark currents $\left(I_{d}\right)$ for films on $\mathrm{Au}$ and on $\mathrm{Al}$ electrodes differed by more than an order of magnitude over a wide range of applied voltages, with much higher currents measured in samples with Au electrodes (Fig. 9). Voltage dependencies of the dark current and of the cw photocurrent observed in samples with $\mathrm{Au}$ and $\mathrm{Al}$ electrodes were drastically different, indicative of different mechanisms responsible for observed currents, depending on the electrode material. In samples with $\mathrm{Au}$ electrodes, the relationship between dark current and voltage $\left(I_{d} \sim V^{\alpha_{d}}\right)$ was close to linear at very low voltages and close to quadratic at higher voltages (e.g. $\alpha_{d}=1.09 \pm 0.03$ at voltages below $2 \mathrm{~V}$ and $1.830 \pm 0.006$ from 2 to $300 \mathrm{~V}$ in an ADT-TES-F film in Fig. 9(a)). In contrast, in samples with $\mathrm{Al}$ electrodes, dark current was weakly voltage-dependent at lower voltages, followed by a steep increase at higher voltages. Despite this sharp increase, even at the highest applied voltage of $300 \mathrm{~V}$ used in our experiments, dark current in samples with $\mathrm{Al}$ electrodes was much lower than that in samples with $\mathrm{Au}$ electrodes at the same voltage. In films on either $\mathrm{Au}$ or $\mathrm{Al}$ electrodes, the $\mathrm{cw}$ photocurrent was higher than the dark current in the entire range of light intensities used. Regardless of the material, in samples with $\mathrm{Au}$ electrodes, $\alpha_{c w}$ obtained from the fit of voltage dependence of the photocurrent $\left(I_{c w} \sim V^{\alpha_{c w}}\right)$ ranged between 1.5 and 2.2, depending on the sample. In any given sample, however, a single value of $\alpha_{c w}$ was sufficient to describe voltage dependence of the $c w$ photocurrent over a large voltage range (e.g. $\alpha_{c w}=1.62 \pm 0.02$ from 0.5 to $100 \mathrm{~V}$ in an ADT-TES-F film in Fig. 9(b)). In samples with Al electrodes, however, $\alpha_{c w}$ was $\sim 1.3-3$ at lower voltages (e.g. $\alpha_{c w}=1.32 \pm 0.05$ in Fig. $9(\mathrm{~b})$ ), depending on the sample, followed by steep transition described by $\alpha_{c w} \sim 3.3-5$ (e.g. $\alpha_{c w}=4.6 \pm 0.2$ in Fig. 9(b)) at higher voltages. Similar trends were observed in ADT-TIPS-F films. ${ }^{11}$

The photoresponse under $\mathrm{cw}$ illumination is strongly affected by the conditions imposed by the electrode 

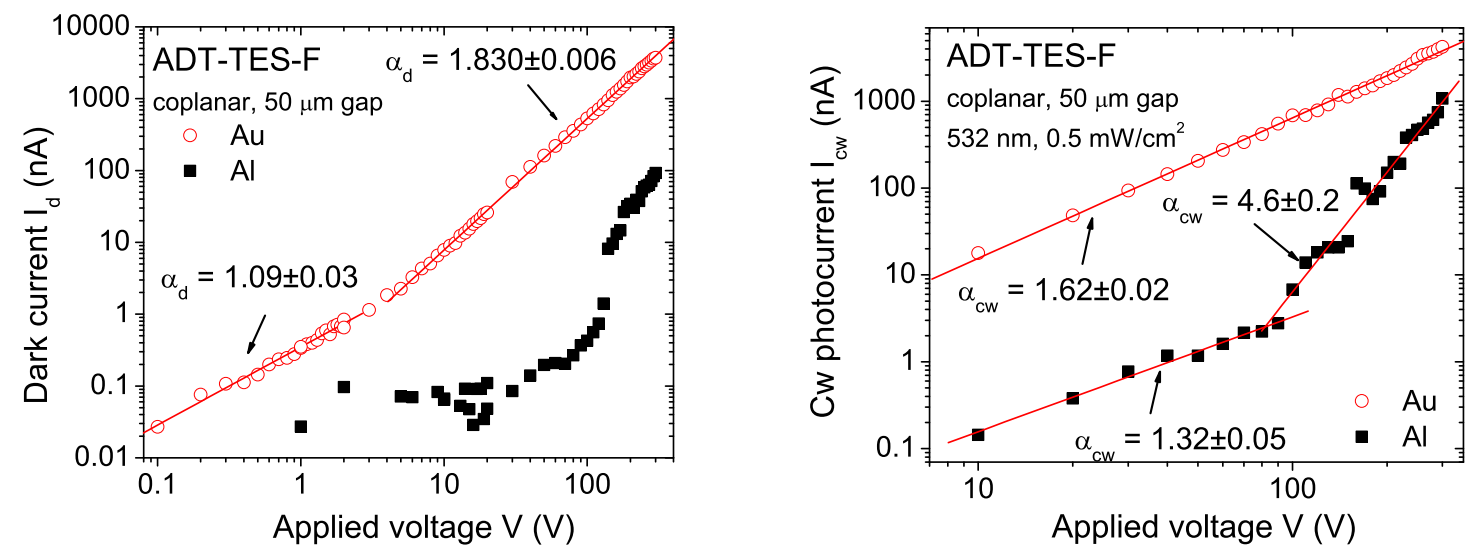

Figure 9. (a) Dark current $\left(I_{d}\right)$ and (b) cw photocurrent $\left(I_{c w}\right)$ obtained in ADT-TES-F films with Al and Au contacts in coplanar geometry with 50- $\mathrm{m}$ gap. Power-law fits $I_{d} \sim V^{\alpha_{d}}$ and $I_{c w} \sim V^{\alpha_{c w}}$ in (a) and (b), respectively, are also shown.

material (Fig. 9(b)) because the steady-state photocurrent $\left(I_{c w}\right)$ is proportional to the photoconductive gain $(G)$, which is much larger than 1 in our samples on Au electrodes (Table 2) due to long carrier lifetimes. In samples with non-injecting electrodes, such as ADT films on Al electrodes at low voltages, the maximal achievable gain cannot exceed $\eta_{0}\left(\eta_{0}<1\right)$ and therefore, the cw photocurrent $I_{c w}$ is much lower. ${ }^{11}$ This is in contrast to the transient photocurrent, the amplitude of which does not significantly depend on the carrier lifetime, thus leading to comparable transient photoresponse of films on $\mathrm{Au}$ and $\mathrm{Al}$ electrodes.

\section{CONCLUSIONS AND OUTLOOK}

In summary, we presented fluorescent and photoconductive properties of functionalized pentacene and ADT derivatives. Single exponential fluorescence decays with lifetimes $\tau$ between 9 and 13 ns and QYs of $\sim 70-75 \%$, depending on the molecule, were observed in toluene solutions of ADT-TES(TIPS)-F, ADT-TIPS-CN, and TIPS pentacene. High fluorescence quantum yields and good stability allowed us to image ADT derivatives on a single molecule level at room temperature at $532 \mathrm{~nm}$ excitation. In films, bi-exponential fluorescence decays were obtained, and fluorescence QYs were reduced to $\sim 40-50 \%$ in ADT-TES(TIPS)-F and to $<0.5 \%$ in TIPS pentacene.

Effective SCLC mobilities $\mu_{\text {eff }}$ of $\sim 0.03-0.092 \mathrm{~cm}^{2} /$ Vs were obtained in ADT-TES-F films. On average, $\mu_{\mathrm{eff}}$ in ADT-TIPS-F and TIPS pentacene films were a factor of $\sim 3$ and $\sim 7$ lower, respectively, as compared to that in ADT-TES-F films. In all films, fast, sub-30 ps charge photogeneration, limited by the time resolution of our setup, and power-law decay of the transient photocurrent $\left(I \sim t^{-\beta}\right)$ with $\beta \sim 0.2-0.6$, depending on the material, were observed. $\mathrm{Cw}$ photocurrents with densities of $\sim 1 \mathrm{~A} / \mathrm{cm}^{2}$ and photoconductive gains of up to 130 were achieved in ADT-TES-F films at $532 \mathrm{~nm}$ photoexcitation with light intensity of $0.58 \mathrm{~mW} / \mathrm{cm}^{2}$ at an average electric field of $4 \times 10^{4} \mathrm{~V} / \mathrm{cm}$. We also examined contribution of effects at metal-organic interfaces to photoresponse. On picosecond time scales, the photoresponse was comparable in films on $\mathrm{Au}$ and $\mathrm{Al}$ electrodes. In samples with $\mathrm{Au}$ electrodes, however, fast initial decay of the transient photocurrent was not as pronounced as in those with $\mathrm{Al}$ electrodes, due to high density of traps filled by holes injected from the Au electrode, which reduced initial trapping of photogenerated holes. The slower decay component of the transient photocurrent, which lasted up to at least microsecond time scales, was not significantly affected by the trapped charge carriers. Dark current and cw photocurrent were higher by more than an order of magnitude in samples on Au electrodes compared to similar films on $\mathrm{Al}$ electrodes.

Availability of several high-performance solution-processable derivatives such as compounds considered here opens up new possibilities to tune optical and electronic properties of materials for specific applications by 
creating mixtures of various derivatives. ${ }^{21,24}$ Our ability to image ADT derivatives on a single molecule level represents an exciting development that allows us to study charge- and energy transfer on a molecular level. This enables comparison between molecular properties and those of bulk films in technologically relevant materials. These studies are currently underway.

\section{ACKNOWLEDGEMENTS}

This work was supported in part by the Petroleum Research Fund, Office of Naval Research (grant \#N0001407-1-0457 via ONAMI Nanometrology and Nanoelectronics Initiative), and National Science Foundation via CAREER program (DMR-0748671).

\section{REFERENCES}

[1] Forrest, S. R., "The path to ubiquitous and low-cost organic electronic appliances on plastic," Nature 428, 911 (2004).

[2] Murphy, A. R. and Frechet, J. M. J., "Organic semiconducting oligomers for use in thin film transistors," Chem. Rev. 107, 1066-1096 (2007).

[3] Geffroy, B., le Roy, P., and Prat, C., "Organic light-emitting diode (OLED) technology: materials, devices and display technologies," Poly. Int. 55, 572-582 (2006).

[4] Ostroverkhova, O. and Moerner, W. E., "Organic photorefractives: mechanisms, materials and applications," Chem. Rev. 104(7), 3267-3314 (2004).

[5] Anthony, J. E., "Functionalized acenes and heteroacenes for organic electronics," Chem. Rev. 106, 50285048 (2006).

[6] Park, S. K., Mourey, D. A., Subramanian, S., Anthony, J. E., and Jackson, T. N., "High-mobility spin-cast organic thin film transistors," Appl. Phys. Lett. 93, 043301 (2008).

[7] Gundlach, D. J., Royer, J. E., Park, S. K., Subramanian, S., Jurchescu, O. D., Hamadani, B. H., Moad, A. J., Kline, R. J., Teague, L. C., Kirillov, O., Richter, C. A., Kushmerick, J. G., Richter, L. J., Parkin, S. R., Jackson, T. N., and Anthony, J. E., "Contact-induced crystallinity for high-performance soluble acene-based transistors and circuits," Nat. Mat. 7, 216 (Mar. 2008).

[8] Ostroverkhova, O., Cooke, D. G., Shcherbyna, S., R. F. Egerton, R. F., Hegmann, F. A., Tykwinski, R. R., and Anthony, J. E., "Band-like transport in pentacene and functionalized pentacene thin films revealed by transient photoconductivity," Phys. Rev. B 71, 035204 (2005).

[9] Ostroverkhova, O., Shcherbyna, S., Cooke, D. G., Egerton, R. F., Hegmann, F. A., Tykwinski, R. R., Parkin, S. R., and Anthony, J. E., "Optical and transient photoconductive properties of pentacene and functionalized pentacene thin films: Dependence on film morphology," J. Appl. Phys. 98, 033701 (2005).

[10] Day, J., Subramanian, S., Anthony, J. E., Lu, Z., Twieg, R. J., and Ostroverkhova, O., "Photoconductivity in organic thin films: From picoseconds to seconds after excitation," J. Appl. Phys. 103, 123715 (2008).

[11] Day, J., Platt, A. D., Subramanian, S., Anthony, J. E., and Ostroverkhova, O., "Influence of organic semiconductor-metal interfaces on the photoresponse of functionalized anthradithiophene thin films," $J$. Appl. Phys. 105, 103703 (2009).

[12] Lehnherr, D., Gao, J., Hegmann, F. A., and Tykwinski, R. R., "Synthesis and electronic properties of conjugated pentacene dimers," Org. Lett. 10(21), 4779-4782 (2008).

[13] Gao, J. and Hegmann, F. A., "Bulk photoconductive gain in pentacene," Appl. Phys. Lett. 93, 223306 (2008).

[14] Wolak, M. A., Delcamp, J., Landis, C. A., Lane, P. A., Anthony, J., and Kafafi, Z., "High-performance organic light-emitting diodes based on dioxolane-substituted pentacene derivatives," Adv. Funct. Mat. 16, 1943-1949 (2006).

[15] Platt, A. D., Day, J., Subramanian, S., Anthony, J. E., and Ostroverkhova, O., "Optical, fluorescent, and (photo)conductive properties of high-performance functionalized pentacene and anthradithiophene derivatives," J. Phys. Chem. C (In Press).

[16] Park, S. K., Jackson, T. N., Anthony, J. E., and Mourey, D. A., "High mobility solution processed 6,13bis(triisopropyl-silylethynyl) pentacene organic thin film transistors," Appl. Phys. Lett. 91, 063514 (2007). 
[17] Subramanian, S., Park, S. K., Parkin, S. R., Podzorov, V., Jackson, T. N., and Anthony, J. E., "Chromophore flourination enhances crystallization and stability of soluble anthradithiophene semiconductors," J. Am. Chem. Soc. 130, 2706 (2008).

[18] Lakowicz, J. R., [Principles of Fluorescence Spectroscopy], Springer, New York (2006).

[19] Lord, S. J., Lu, Z., Wang, H., Willets, K. A., Schuck, P. J., Lee, H.-l. D., Nishimura, S. Y., Twieg, R. J., and Moerner, W. E., "Photophysical properties of acene DCDHF fluorophores: Long-wavelength single-molecule emitters designed for cellular imaging," J. Phys. Chem. A 111, 8934-8941 (2007).

[20] Haugland, R. P., ed., [The Handbook: A Guide to Fluorescent Probes and Labeling Technologies], Molecular Probes, Eugene, OR, 10 ed. (2005).

[21] Day, J., Platt, A. D., Ostroverkhova, O., Subramanian, S., and Anthony, J. E., "Organic semiconductor composites: influence of additives on the transient photocurrent," Appl. Phys. Lett. 94, 013306 (2009).

[22] Coppo, P. and Yeates, S. G., "Shining Light on a Pentacene Derivative: The Role of Photoinduced Cycloadditions," Adv. Mat. 17, 3001-3005 (2005).

[23] Bässler, H. and Schweitzer, B., "Site-selective fluorescence spectroscopy of conjugated polymers and oligomers," Acc. Chem. Res. 32, 173-182 (1999).

[24] Platt, A. D., Shepherd, W. E. B., Anthony, J. E., and Ostroverkhova, O., "Photophysical and photoconductive properties of organic semiconductor composites," Proc. SPIE $\mathbf{7 4 1 3}$ (2009).

[25] Pope, M. and Swenberg, C. E., [Electronic Processes in Organic Crystals], Oxford University Press, New York (1982).

[26] Como, E. D., Loi, M. A., Murgia, M., Zamboni, R., and Muccini, M., "J-aggregation in $\alpha$-sexithiophene submonolayer films on silicon dioxide," J. Am. Chem. Soc. 128, 4277-4281 (2006).

[27] der Auweraer, M. V. and Scheblykin, I., "One-dimensional J-aggregates: Dependence of the properties of the exciton band on the model of the intermolecular coupling," Chem. Phys. 275, 285-306 (2002).

[28] Spano, F. C., "Temperature dependent exciton emission from herringbone aggregates of conjugated oligomers," J. Chem. Phys. 120, 7643-7658 (2004).

[29] Platt, A. D., Day, J., Shepherd, W. E., and Ostroverkhova, O., "Photophysical and photoconductive properties of novel organic semiconductors," in [ACS Organic Thin Films for Photonic Applications], American Chemical Society, Washington D.C. (2009).

[30] Ostroverkhova, O., Cooke, D. G., Hegmann, F. A., Anthony, J. E., Podzorov, V., Gershenson, M. E., Jurchescu, O. D., and Palstra, T. T. M., "Ultrafast carrier dynamics in pentacene, functionalized pentacene, tetracene and rubrene single crystals," Appl. Phys. Lett. 88, 162101 (2006).

[31] Moses, D., Soci, C., Chi, X., and Ramirez, A. P., "Mechanism of carrier photogeneration and carrier transport in molecular crystal tetracene," Phys. Rev. Lett. 97, 067401 (2006).

[32] Hu, W., Pflaum, J., Schweitzer, D., and Dressel, M., "Transport properties of [2,2]-paracyclophane thin films," Appl. Phys. Lett. 84, 4720-4722 (2004).

[33] Day, J., Platt, A. D., Subramanian, S., Anthony, J. E., and Ostroverkhova, O., "Influence of organic semiconductor-metal interfaces on the photoresponse of functionalized anthradithiophene thin films," $J$. Appl. Phys. 105, 103703 (2009).

[34] Jurchescu, O. D., Subramanian, S., Kline, R. J., Hudson, S. D., Anthony, J. E., Jackson, T. N., and Gundlach, D. J., "Organic single-crystal field-effect transistors of a soluble anthradithiophene," Chem. Mat. 20(21), 6733-6737 (2008).

[35] Lee, C., Yu, G., Moses, D., Pakbaz, K., Zhang, C., Sariciftci, N. S., Heeger, A. J., and Wudl, F., "Sensitization of the photoconductivity of conducting polymers by C60: Photoinduced electron transfer," Phys. Rev. B 48, 15425 (1993).

[36] Godlewski, J., Jarosz, G., and Signerski, R., "Photoenhanced current in thin organic layers," Appl. Surf. Sci. 175-176, 344-350 (2001).

[37] Itaka, K., Myojin, N., Yamashiro, M., Yamaguchi, J., and Koinuma, H., "Molecular beam epitaxy of highly oriented pentacene thin films on an atomically flat sapphire substrate," Jap. J. Appl. Phys. 44, 6249-6251 (2005). 